\title{
HABILIDADES DE PENSAMIENTO SIMBÓLICO: URDIMBRES DE SIGNIFICADO, SOCIEDAD Y TIC
}

\author{
Miguel A. Romero Morett ${ }^{1}$ \\ Universidad de Guadalajara - México \\ miguelromeromorett@gmail.com \\ Aracely Forero Romero ${ }^{2}$ \\ Universidad Pedagógica y Tecnológica de Colombia - Colombia \\ Grupo de Investigación HISULA \\ aracely.forero@uptc.edu.co \\ Alfredo Cedano Rodríguez ${ }^{3}$ \\ Universidad de Guadalajara - México \\ alfredo.cedano@cutonala.udg.mx
}

Recepción: 20/06/2012

Evaluación: 29/09/2012

Aceptación: 15/11/2012

Artículo de Revisión

doi: http://dx.doi.org/10.9757/Rhela.1905

\section{RESUMEN}

El objetivo y el contenido de esta intervención radica en problematizar la construcción de pensamiento simbólico en el contexto de una sociedad definida por las Tecnologías de la Información y la Comunicación que, a su vez, configuran la actual identidad y vocación de la colectividad humana, creadora, de suyo y por derecho pro- pio, de las cargas de significado y de creación de sentido de los acontecimientos humanos y de los fenómenos naturales. Particularmente el acceso a esta problematización tiene como fuente la puesta en relación entre autores, la recuperación de la experiencia docente en la Maestría en Estudios Humanísticos del TEC de Monterrey y en

\footnotetext{
Doctor en Educación de la Universidad La Salle. Maestro en Educación de la Universidad del Valle de Atemajac. Licenciado en Filosofía de la Universidad de Guadalajara. Actualmente jefe del Departamento de Ciencias Sociales del Centro Universitario de Tonalá, profesor investigador del Departamento de Filosofía de la Universidad de Guadalajara y profesor de la Maestría en Humanidades de la Universidad Virtual del TEC de Monterrey. Miembro del cuerpo académico procesos educativos, del Centro Universitario de Ciencias Sociales de la Universidad de Guadalajara. Línea de investigación: desarrollo de habilidades dialógicas y educación.

2 Aplicadas a la Educación. Universidad Pedagógica Nacional Especialista en Gerencia Informática. Universidad Pedagógica y Tecnológica de Colombia. Psicología Universidad de los Andes de Bogotá. Actualmente. Profesora titular de la Universidad Pedagógica y Tecnológica de Colombia. Coordinadora de la red de formación e investigación CAFETIC (Comité Académico para la Formación Educativa en TIC), integrante del grupo de investigación - HISULA.

3 Maestro en Administración de Negocio por la Universidad de Guadalajara. Ingeniero en computación por la Universidad de Guadalajara. Actualmente es Coordinador de Tecnologías para el Aprendizaje del Centro Universitario de Tonalá y profesor del Departamento de Ingenierías y del Departamento de Ciencias Aplicadas a la Información de la Universidad de Guadalajara.
} 
el Departamento de Filosofía de la Universidad de Guadalajara, y la constatación cuantitativa de la importancia del uso de las tecnologías de información y comunicación en México y en el Estado de Jalisco, con lo cual se conforma un triángulo cuyos vértices se remiten recíprocamente.

\section{SYMBOLIC THINKING SKILLS: MEANING WRAPS, SOCIETY AND ICTS}

\section{ABSTRACT}

The objective and content of this intervention has its core in mentioning the problems of the construction of a symbolic thinking in the context of a society defined by ICTs, which at the same time configure the current identity of the human kind, creator and by its own right of the meaning and creation of sense of the human events and natural phenomena. Particularly regarding the Access to this problem mentioning, it has its source in the implementation of the conjunction among authors, the recovery of the teaching experience in the Monterey Master's degree on Humanistic Studies and in the Department of Philosophy at the Universidad de Guadalajara, and the quantitative checking on the importance of the usage of ICTs in Mexico and the state of Alisco, with this a triangle whose corners come in contact with one and other.

Key words: History of Latin American Education Journal, on-line education,symbolic thinking, lineal, mitic, rational, univocal, narrative,cause, thinking structures, right now culture, connectivity and multitask culture, ICT.
Palabras clave: Revista Historia de la Educación Latinoamericana, educación virtual, pensamiento simbólico, lineal, mítico, racional, univoco, narrativo, causal, estructuras de pensamiento, cultura de la inmediatez, de la conectividady de la multitarea, tecnologías de la comunicación y la información.

\section{HABILIDADES DO PENSAMENTO SIMBÓLICO: TECIDOS DE SIGNIFICADO, SOCIEDADE E TICS}

\section{RESUMO}

O objetivo e o conteúdo desta intervenção são a problematização da construção do pensamento simbólico no contexto de uma sociedade definida pelas Tecnologias da Informação e a Comunidade que, por sua vez, configuram a atual identidade e vocação da coletividade humana, criadora, por si e por direito próprio, das cargas de significado e de criação de sentido dos acontecimentos humanos e dos fenômenos naturais. Particularmente o acesso a esta problematização tem como fonte o que está posto em relação entre autores, a recuperação da experiência docente no Mestrado em Estudos Humanísticos do TEC de Monterrey e no Departamento de Filosofia da Universidade de Guadalajara, e a constatação quantitativa da importância do uso das tecnologias de informação e comunicação no México e no Estado de Jalisco, com o qual se conforma um triangulo cujos vértices se remetem reciprocamente.

Palavras-chave: Revista História da Educação Latino-americana, educação virtual, pensamento simbólico, linear, mitico, racional, univoco, narrativo, causal, estruturas de pensamento, cultura do imediatismo, da conectividade e da multitarefa, tecnologias da comunicação $e$ da informação.

\section{INTRODUCCIÓN}

\section{La constatación cuantitativa de internautas}

En la actualidad es imposible imaginarnos una entidad pública o privada que brinde sus servicios a grandes masas de población sin la utilización de las Tecnologías de la Información y la Comunicación (TIC). Internet es un medio no tangible por el cual se comparten altos volúmenes de información y cuya explicación técnica se expresa como una red de computa- 
doras interconectadas para intercambiar datos científicos. Su importancia se resume en los siguientes datos ${ }^{4}$ : en México, existen 40.6 millones de usuarios de Internet y Jalisco cuenta con 3 millones de internautas, solo detrás del Estado de México con más de 6 millones y el Distrito Federal con más de 4 millones (Asociación Mexicana de Internet, 2013, 2011). Según la Asociación Mexicana de Internet, 3 de cada 10 hogares en Jalisco cuentan con una computadora de las cuales el 76\% posee una conexión a Internet. Sin embargo, menos del 3\% de los internautas a pesar de contar con una computadora en su casa destina sus actividades en la red a estudiar. En suma, a las dificultades que se expondrán líneas abajo en torno a la generación de pensamiento fraccionario añadiremos que las potencialidades del internet y de otras formas de TIC se desperdician, incluso en los fondos documentales electrónicos por los que las universidades erogan grandes sumas ${ }^{5}$.

Por otra parte, datos relevantes nos muestran la importancia del correo electrónico y las redes sociales. Las ventajas son tales que cualquier persona es capaz de incorporar a su mensaje cualquier tipo de video, voz, y datos, sin importar la distancia geográfica que separe al emisor del receptor. Con el correo electrónico, la comunicación es más rápida, y aporta elementos para lograr que nuestra sociedad sea sustentable, y lo más importante incrementa la productividad y la eficiencia en donde se tenga implementado este recurso tecnológico si se utiliza de la manera adecuada, ya que permite compartir archivos, e intercambiar ideas sin importar la distancia geográfica que separe a los destinatarios. Este medio es ampliamente empleado en los contextos educativos y, al adquirir el carácter de redes sociales se generan urdimbres de especialistas que compartes datos de carácter científico.

En México existen 25.6 millones de internautas mayores a 6 años en las zonas urbanas, 5 millones en las zonas no urbanas, generando un 32,5\% de tasa de penetración nacional de internet en personas mayores a 6 años

4 Toda la información numérica del presente apartado está tomada de las siguientes referencias: Asociación Mexicana de Internet. Estudios/ Asociación Mexicana ((1 de enero de 2011). Recuperado el 15 octubre de 2011, http://www.amipci.org.mx/temp/Habitos2011AMIPCI-VersionEjecutiva-000834300130679431OB.pdf y de Asociación Mexicana de Internet. (4 de 1 de 2013). Asociación Mexicana de Internet. Obtenido de Asociación Mexicana de Internet: www.amipci.org.mx.

5 Pablo Beneitone, et al. Reflexiones y perspectivas de la educación superior en América Latina. Informe final - Proyecto Tuning - América Latina, 2004-2007 (Espańa: Universidad de Deusto, 2007), 18. 
de edad. En las zonas urbanas el 37\% de hogares cuenta con una computadora personal. De los cuales, 7 de cada 10 accede a internet. Los jóvenes de México son los usuarios más importantes de internet, ya que son usuarios el 68\% dentro del grupo de edades entre los 12 a los 19 años de edad, y en el grupo de edad entre los 25 a los 34 años son únicamente el $38 \%$, sin embargo su tasa de crecimiento es la mayor alcanzando un valor del 34\%. Dentro de las actividades realizadas por los internautas el correo electrónico continúa siendo la principal actividad social on-line con un $75 \%$ de personas que realizan esta actividad, tal como lo revela la Asociación Mexicana de Internet ${ }^{6}$.

Por otra parte, la nube se ha convertido en la tendencia de cómputo con más auge después de las redes sociales y la Web 2.0; en la nube se pueden hacer procesos de cómputo distribuidos y en paralelo lo cual acorta el tiempo de procesamiento. Para el usuario final se generan soluciones de almacenamiento, de tal manera que dispone de sus documentos en cualquier dispositivo simplemente con una conexión a internet. Esta capacidad de acceder a sus documentos y que se encuentren sincronizados, le da al usuario la capacidad de concluir algún trabajo o investigación sin necesidad de transportarlo o enviárselo a sí mismo por correo electrónico para terminarlo en casa ahorrando tiempo y energía del dispositivo desde el cual se consulta ${ }^{7}$.

La virtualización ${ }^{8}$, en otro sentido ha vivido un cambio de significado cuando lo contextualizamos dentro de las TIC, pues ha pasado de ser algo irreal o algo que solo puede existir en un mundo imaginado a ser la digitalización del mundo real, sin importar cuál sea el objeto, las imágenes, los textos y diversas expresiones del saber. Con esta transformación podemos llevar todo lo tangible en algo intangible representado por código binario -la más común de las simbolizaciones en las ciencias asociadas a las TIC-, el cual puede ser almacenado en nuestro bolsillo y pesar menos de 500 gramos ${ }^{9}$. Así en estos días ya se puede virtualizar cualquier cosa, un espa-

6 Instituto Nacional de Estadística y Geografía de México. En México 38.9 millones de personas usan una computadora y 32.8 millones utilizan internet. Obtenido (17 de febrero de 2013). whttp://www.inegi.org.mx/ inegi/contenidos/espanol/prensa/comunicados/modutih10.asp

7 Asociación Mexicana de Internet. Asociación Mexicana de Internet. Obtenido de Asociación Mexicana de Internet, (4 de 2013), www.amipci.org.mx

8 Pierre Lévy, ¿Qué es lo virtual? (Barcelona: Paidós, 1999), 13-15.

9 L. Shulman, Knowledge and teaching: foundations of the new reform. (Harvard Educational Review, 57 (1), 1987), 1-22. 
cio físico o un diseño industrial. Como se aprecia, la virtualización constituye una herramienta de aprendizaje que permea a las ciencias, como la medicina, pues de esa manera se puede diseccionar el cuerpo humano, o bien, la arquitectura, en la medida que se puede visualizar una edificación desde todos los ángulos interiores y exteriores. El uso de esta herramienta ha acrecentado los niveles de simbolización y abstracción de los agentes directos de la educación. Igual que en los anteriores, no se da el caso de que este instrumento se aproveche generalizadamente ${ }^{10}$.

En décadas pasadas, la educación a distancia se impartía por medio de televisiones y señales educativas que eran mostradas a los alumnos sin ningún tipo de interacción entre ellos y su tutor o profesor. Hoy en día la educación a distancia no solo es televisiva, sino impartida por medio de internet y computadoras.

Particularmente la educación a distancia ha cobrado una valía insospechada a causa de que las plataformas virtuales, como Moodle, Blackboard, WebCT (las cuales pueden ser tanto de software libre como de software propietario), han ido evolucionando para brindar espacios y comunidades virtuales, las cuales brindan a los alumnos no solo un aula virtual, sino también la posibilidad de tener laboratorios virtuales, en los cuales se pueden hacer prácticas de los conocimientos adquiridos en las clases de teoría. De la misma manera, los estudiantes pueden acceder a las bibliotecas virtuales, donde existen numerosos catálogos de libros disponibles para consultar y complementar la información y enseñanza recibida en las clases a distancia. El dominio de la educación a distancia ${ }^{11}$ consiste en el conocimiento de la semiótica que las plataformas emplean, la cual no se reduce en absoluto a los íconos que anteceden las múltiples operaciones, como el acceso a un menú de posibilidades, sino al aprendizaje de la lógica cibernética conforme proceden las herramientas situadas en el equipo y los programas.

Existen otras herramientas, las cuales no son exactamente plataformas de educación a distancia, que sin embargo facilitan el proceso de ense

10 Rubén B. D., En la era de la información: información, tecnología y estudio del comportamiento. (Rutgers University. New Jsersey, Rutgers University, 2005), 53-72.

11 Donna Zapata Zapata, Contextualización de la enseñanza virtual en la educación superior. (Bogotá: ICFES, 2002). http://blade1.uniquindio.edu.co/uniquindio/ntic/lineamientos/nuevos\%20libros/arc_88.pdf (21 de Mayo, 2012). 
ñanza-aprendizaje que las instituciones educativas requieren. Estas herramientas son YouTube, Facebook, Twitter, Slideshare, Google Hangout, algunas de las cuales son estrictamente redes sociales, pero al permitir el intercambio masivo de ideas, documentos o información a través de medios digitales, permiten el proceso de enseñanza a distancia que tanto se busca. Además, estas herramientas son de uso cotidiano por los jóvenes entre 14 a 22 años, los cuales se encuentran entre los rangos de edades de estudiantes potenciales.

Estas edades y aún las más tempranas, constituyen escenarios de tiempo en los que la semiótica de la operatividad de los equipos y programas facilita a los iniciados o dificulta a los legos, el conocimiento especializado en las tecnologías en cuestión. Igual que en los casos anteriores, la educación a distancia, el uso de plataformas, la creación de blogs y páginas Web, el manejo del correo electrónico, la incorporación a las redes sociales, el conocimiento de los programas de virtualización de la realidad fuera de la conciencia, conforman una experiencia cotidiana de creación simbólica, de deconstrucción, de acercamiento con extrañeza al mundo y a los sistemas de signos y, en suma, conviene al aprendizaje significativo. Así, el acercamiento simbólico y la toma de conciencia de la significación semiótica del mundo vienen a la par del dominio de competencias simbólicas. Continuemos pues, la problemática simbólica asociada a las TIC $^{12}$.

\section{E1 pensamiento simbólico}

Como pensamiento simbólico se reconoce específicamente la expresión que mediatiza la realidad mediante signos, símbolos, signifi-cados, significantes, imágenes acústicas, fonemas o cualquier otro término, propios de las diversas teorías lingüísticas, semiológicas o de la comunicación y donde, como formas simbólicas particulares figuran las metáforas y los aforismos. La condición del pensamiento simbólico consiste, en cualquiera de los casos, en establecer ligas y mediaciones de comprensión de la realidad-aquello que de alguna manera figura como objeto en sí mismo de aprehensión y cognición ${ }^{13}$, y la conciencia, vinculación que se enmarca en las operaciones de pensamiento ${ }^{14}$.

12 Jonathan Lomelí López, “De la letra impresa al byte. ¿'Internet está reprogramando nuestro cerebro?”, Revista del ITESO. Diciembre 2012 - Enero 2013/ Año XLII, No 431, (2011): 38-44.

13 Jost Herbig, La evolución del conocimiento cientifico. Del pensamiento mitico al pensamiento racional (Barcelona: Herder, 1997), 19-49.

14 Roger Bartra, Antropología del cerebro. La conciencia y los sistemas simbólicos. (México: FCE, 2012), 37-56. 
Como es natural, el pensamiento simbólico es una forma -tal vez la más fina- del pensamiento abstracto, aquel que busca patrones comunes de significados entre aspectos de realidades heterogéneas, y que posee diversas manifestaciones, como la forma de pensamiento teórico - abstracto, creativo - innovador y causal. Aunque habitualmente lo oponemos al pensamiento abstracto, el pensamiento plástico - descriptivo, pese a su expresión material y sensorial, posee la posibilidad de conformar y proyectar las estructuras cognitivas de mayor complejidad. El pensamiento simbólico puede ser ubicado entre ambos, sin que por ello, exista el riesgo de equivocidad en el significado. Junto a estas formas de pensamiento figuran aquellas que por razones de otras especificidades como el carácter formal -en el caso de la lógica y la matemática- o el carácter comparativo entre niveles distintos de significado -como en el caso del pensamiento analógico- se asocian a la ciencia y a la poesía. Merece, para efectos de la presente reflexión, detener la atención en las formas de narración -dicho no en su restricción literaria, sino en su amplitud de concatenación de historias, argumentos y procesos de inferencia.

\section{El pensamiento causal en el contexto de la Cultura}

La segunda parte de la vinculación propuesta figura en la cultura, es decir, en la sociedad misma desde el punto de vista del significado y de la construcción colectiva del sentido, en la historia humana y la historia natural. El punto de partida de la reflexión en este apartado radica en los acontecimientos y los fenómenos son de por sí, silentes y secretos y que para comprender qué es lo que figura detrás de las expresiones puramente fenoménicas de la realidad, es obligado buscar las urdimbres de causalidades, a la vez, diseñar el sentido con expectativas teleológicas que le atribuimos a la historia de las sociedades. En la búsqueda de significados de la sociedad y en la atribución de sentido a la historia figura aquello que nos salva del caos de lo sensible y nos habilita para comprender, aprehender, significar e intervenir sobre lo empírico, las categorías de espacio y tiempo y las cualidades de la substancia, lo que podemos asumir como la dimensión conceptual que confiere inteligibilidad a la realidad errática. Dicho de manera coloquial, sin conceptos no comprendemos la empiria del mundo o de la vida cotidiana ${ }^{15}$, ni los fenómenos naturales ni los problemas de las

15 Edgar Morin, El Método II. La vida de la Vida. (Madrid: Ediciones Cátedra, 1997), 234-242. 
ciencias. Siendo así, para los efectos de los procesos educativos el ejercicio de simbolización y deconstrucción de significados, de conformación y metaconformación de estructuras cognitivas ${ }^{16}$ en los procesos educativos habituales constituye un pendiente sobre el que debemos investigar, por la vía de un acercamiento con extrañeza o por la vía de la investigación cuantitativa. Para completar la digresión formulo una pregunta acuciante: ¿Cómo formar ciudadanos que vivan conforme a la justicia y el derecho, solidarios e integrados, si sus procesos cognitivos y sus estructuras de pensamiento están distorsionadas? ${ }^{17}$.

\title{
3. Pensamiento lineal
}

Para efectuar el presente acercamiento a la temática expuesta tomo como punto de partida el rescate de la experiencia docente en cursos presenciales en pregrado, posgrado y en línea en ámbitos cognitivos de carácter filosófico. El problema se resume en la oposición entre el pensamiento secuenciado y el pensamiento de saltos. El primero se refiere al pensamiento estructurado, ordenado, lineal, con base en argumentación y sentido lógico, si bien con los limitantes de las propias fronteras argumentativas que impide excesos informativos, "bandazos" narrativos y añadidos creativos. En frase de Richard Watson, en su libro Mentes del futuro:

\begin{abstract}
La forma correcta de razonar -lo que llamo pensamiento profundo- es lo que nos convierte realmente en humanos. Este es el tipo de pensamiento que está relacionado con las ideas nuevas que hacen avanzar el mundo, el tipo de razonamiento inherente a la planeación estratégica, al descubrimiento científico y a la invención artística. Se trata de un pensamiento que es riguroso, centrado, deliberativo, considerado, independiente, original, imaginativo, ancho, amplio, tranquilo, relajado, atento, contemplativo y reflexivo, en el que el flujo de información es limitado y los medios cuentan: lo que se podría llamar «flujo lento», fracturado o distraido ${ }^{18}$.
\end{abstract}

Como inmediatamente se aprecia, este es el tipo de pensamiento en el que se empeña la educación tradicional, escolarizada, ubicada en la cultura de la idea y de la lectura. Impulsar el pensamiento profundo no es nada fácil y lo es aún menos en el contexto de la sociedad identificada y operada

16 B. Honoré, Para una teoría de la formación. (Madrid: Narcea, 1980), 26 y 49.

17 Miguel A. Romero Morett, Desarrollo de habilidades filosóficas. Un estudio comparativo y transdisciplinar en el campo educativo. (México: Universidad de Guadalajara, 2006), 3-8.

18 Richard Watson, Mentes del futuro. ¿Está cambiando la era digital nuestras mentes? (Barcelona: Viceversa ayer y hoy, 2011), 13. 
Habilidades de pensamiento simbólico: urdimbres

de significado, sociedad y TIC

por las tecnologías de la comunicación y la información. Particularmente, en los cursos en línea superar el pensamiento simple y simplificador es un reto que enfrenta el diseño instruccional y, junto a ello, la conformación de estructuras cognitivas de carácter analítico. Jonathan Lomelí López, editor del portal de noticias e investigador de la comunicación digital, reconoce justamente que:

Estamos ante una transición en nuestra historia intelectual y cultural: se confrontan dos modelos de pensamiento. El viejo pensamiento lineal y el nuevo pensamiento no lineal. El primero implica concentración, atención a una sola cosa a la vez, principio y final. El pensamiento no lineal que representa internet tiene avidez por absorber y compartir información atomizada, inconexa e inmediata: entre mayor rapidez (y más), mejor. Infinitud e instantaneidad. Ahora y todo. El pensamiento lineal es el pensamiento gutenberiano. Es el libro. La mente literaria y reflexiva. La que guió y dio cauce al Renacimiento, la Ilustración, la Revolución Industrial, pero que probablemente encuentra su punto más crítico y su disolución con internet ${ }^{19}$.

A diferencia de la antigua educación lineal, que impulsa el pensamiento porpasos, la educación en la virtualidad procede mediante lo que podríamos denominar lúdicamente, educación de saltamontes.

\section{Pensamiento por saltos y las formas narrativas}

El pensamiento por saltos está confeccionado a la manera de ramas sobre las cuales la narración, la descripción y la argumentación se expanden, con los riesgos naturales del extravío, si bien con las posibilidades de la amplitud informativa, no necesariamente procesada ${ }^{20}$. En la base de esta oposición figuran nuevas formas narrativas que, a la par de la literatura, en el ensayo filosófico y en los reportes de investigación sociológica, tienen presencia. Tomo como ejemplo la película mexicana Babel, del cineasta Alejandro González Iñárritu, de la cual hago una breve reseña en atención a quienes no la hubiesen visto:

En Marruecos, un pastor compra un rifle Winschester calibre. 270 y lo entrega a sus dos bijos, Yussef y Ahmed, con el objeto de que abuyenten a los depredadores que amenazan el rebaño. Probándose uno al otro, efectúan disparos, uno de los cuáles es dirigido inten-

19 Jonathan, Lomelí López, “De la letra impresa al byte. ¿Internet está reprogramando nuestro cerebro?” Magis. Revista del ITESO. Diciembre 2012 - Enero 2013/ Año XLII, No. 431 (2011), 42.

20 Luis Armando Aguilar et al., Lo humano en riesgo. Educación frente a la globalización. Instituto tecnológico de Occidente. (México, Publicaciones Itseo, 2005), 19-20. 


\begin{abstract}
cionalmente a un camión de pasajeros, considerado tan sólo como objeto móvil. La bala biere a Susan, quien viaja con su esposo Richard, con quien guarda una relación muy deteriorada; de hecho, el viaje podía entenderse como un intento fallido de reencuentro. Mientras tanto, los bijos de ambos permanecian en California bajo el cuidado de una niñera mexicana, Amelia, cuyo bijo se casaría en Tijuana, en unos dias. Amelia pasa la frontera llevándose a los niños, pues ni puede dejar de acudir a la boda ni podía dejar que los niños se quedasen solos. Una vez realizada la boda, su sobrino Santiago los conduce de nuevo a casa, sólo que despierta sospechas en el cruce fronterizo. Santiago, ebrio e impulsivo, desafía a los guardias, huye y se interna en el desierto, entre la angustia de Amelia y los niños, que no comprenden nada. Por otra parte, en el Japón, Chieko, una adolescente sordomuda y rebelde, guarda muy mala relación con su padre, Jasijuro, quien es buscado por la policía para que explique la posesión del rifle con el que Susan fue berida ${ }^{21}$.
\end{abstract}

El punto de partida de la historia fue el disparo, lo que entrelazó historias en tres continentes, en tres escenarios naturales y culturales diferentes, con personajes marcadamente distintos y complejos, tejiendo una red de causalidades asociadas, no secuenciadas de manera lineal. Con esta y otras formas narrativas se van modelando nuestras formas de percibir y referir la realidad. En veces partimos del desenlace y nos remitimos retrospectivamente con el objeto de esclarecer las causas y circunstancias preliminares; otras veces hacemos caminos de ida y vuelta, adelantando o rezagando la historia y dejando que el lector o el espectador la re-construya ${ }^{22}$.

Como se aprecia, hoy tenemos modelos narrativos que se prestan a múltiples conclusiones o que dejan abierta la historia para que el lector o el espectador la complementen a su arbitrio. En suma, en literatura hace mucho quedó superada la narración lineal, eslabonada, en la que había un inicio, un desarrollo y una conclusión. El aprendizaje en la virtualidad es un aprendiaje por saltos, en la medida que está saturada de hipertextos y que, en la búsqueda de significaciones semánticas o históricas caemos en el extravío de la ruta que parece carecer de camino de regreso al punto de partida, o en el extravío del exceso de información, sobre la que pesa la superficialidad de los datos de las primeras búsquedas, la dificultad para seleccionar entre textos sustentados y fiables y documentos de pura opinión; o bien, entre niveles de profundidad y densidad en contenidos y de rigurosidad y lógica en la argumentación.

21 Alejandro González Iñárritu, La torre de Babel. Fragmento de película 2006.

22 Richard Watson, Mentes del futuro. ¿Está cambiando la era digital nuestras mentes? Viceversa ayer y hoy, (Barcelona: Viceversa ayer y hoy, 2011), 23. 
Habilidades de pensamiento simbólico: urdimbres

de significado, sociedad y TIC

En el plano argumentativo estamos adheridos a los estilos narrativos, algunos de carácter lineal, como los propios de la lógica clásica, de origen aristotélico, o la lógica matemática en sus diversas formas, por ejemplo, proposicional o cuantitativa, en donde las premisas obligan a una conclusión y donde el cuidado de no caer en las falacias es extremo. En esta forma argumentativa desde el título de un ensayo se prevé el universo de análisis, el acercamiento metodológico y el contexto. Además, las digresiones son mal vistas y entorpecen la ruta argumentativa y narrativa ${ }^{23}$.

\section{5. ¿Generan las TIC nuevas estructuras e inferencias de pensamiento?}

Es clara la aplicación de la lógica a la ciencia, por ejemplo, a través de la contrastación y la falsación, pero no necesariamente es una lógica que se aplica a la vida cotidiana o a la resolución de problemas de orden práctico. De ahí que surja la cuestión si esta lógica narrativa ha dejado de tener aplicación en el aprendizaje contextualizado en las actuales tecnologías de la comunicación y la información. ¿Pensamos hoy a la manera de Babel, por detonantes argumentativos? ¿Pensamos, no en secuencias, sino en redes, en estructuras cognitivas conformadas por arborizaciones, por escenarios múltiples, por saltos, por links o por ventanas, por esquemas de posibilidades, por lógicas no bivalentes, sino polivalentes o peor aún, por ambivalencias o por equivocidades? ¿No se da el caso de que -dicho en el otro sentido, el originario, el bíblico- enfrentamos sistemas de signos y símbolos, de lenguajes de computación, de iconografías o de plásticas que no comprendemos y nos alejan, y cuyas decodificaciones reclaman una permanente atención a la forma, dejando de lado el contenido? ¿No es hoy más cierto que nunca el mensaje de Marshall McLuhan, en el medio está el mensaje o mejor, en el significante - la expresión material del signo- está el mensaje, en el fonema está el mensaje o peor aún, en el hardware está el mensaje? ${ }^{24,25}$.

Así dicho, de inicio parece que la simbólica del hardware y el software configuran la identidad y la vocación del hombre; pero parece, además que, fuera del valor sacralizado de la tecnología solo existen connotaciones

23 Blanca Solares (coord.), Los lenguajes del símbolo. Investigaciones de hermenéutica simbólica. (México: Anthropos UNAM, 2011), 1-13.

24 Marshall Mcluhan, La Aldea. Global. Baricco, Alessandro. $1^{\text {a }}$ edición, (México, 1991), 203.

25 ¿Qué significado se transmite mejor, el del nivel económico de quien porta un teléfono inteligente de última generación o bien los mensajes escritos, orales, gráficos o simbólicos que su poseedor recibe y transmite? 
negativas, por lo menos para quienes han sido formados en la cultura de la idea y llegaron tarde a la cultura de la imagen ${ }^{26}$, del almacenamiento y procesamiento de información y al envío inmediato de datos. Sin embargo, la cuestión que merece atención podría expresarse en términos de la parte irremplazable del aprendizaje sustentado en las tecnologías de información y comunicación que conducen a la apertura de mente, a la creatividad y al acceso a la información ${ }^{27}$.

Hago referencia a un autor que ya aborda el tema y que por ello ofrece la posibilidad de contrastar su planteamiento con la experiencia docente cotidiana y abre horizontes ${ }^{28}$ de investigación. Me refiero a Richard Watson, en su libro Mentes del futuro. Para ser congruente con el pensamiento secuencial su subtítulo resume la pregunta que permea toda su disertación: ¿Está cambiando la era digital nuestras mentes?, lo que traduciríamos en un doble sentido: el cambio bioneuronal de la corteza cerebral y el cambio en la conformación de estructuras cognitivas con las que aprehendemos el mundo y a nosotros mismos.

Watson describe los contextos de la vida digital y recupera la idea de un autor, Michael Merzenich, "un neurocirujano pionero que descubrió a través de experimentos que el cerebro humano es «plástico»: responde a cualquier estímulo o experimento nuevos. Por ello nuestro pensamiento está enmarcado por las herramientas que decidimos usar... provocando que se remodele a profundidad" (Watson) ${ }^{29}$. En consonancia con ello, Watson recuerda que "ya estamos tan conectados a través de las redes digitales que se ha desarrollado una cultura de la respuesta rápida"30. Considerando estas dos ideas, es fácil reconocer que algo está ocurriendo en nuestras estructuras cognitivas, aquellas que, desde una perspectiva clásica se empeñaba por reconocer lo que es en sí y distinguirlo de lo que es en otro, a la manera aristotélica. Sin embargo, ya la mente duda, pues la virtualidad se entreteje con la realidad y esta ha dejado de poseer su propia onticidad, para convertirse en una construcción de la mente, donde lo que es, se entrelaza

26 José Luis Brea. (Editor). Estudios visuales. La epistemología de la visualidad en la era de la globalización. (Madrid: Akal, 2005), 2.

27 Lévy, Pierre. ¿Qué es lo virtual? (Barcelona: Paidós, 1999), 13-15.

28 Carlos Reynoso. Corrientes teóricas en antropología. Perspectivas desde el siglo XXI. (Buenos Aires: El Argonauta, Colección de Complejidad Humana, 2008), 264.

29 Richard Watson, Mentes del futuro, 12.

30 Richard Watson, Mentes del futuro, 12. 
Habilidades de pensamiento simbólico: urdimbres

de significado, sociedad y TIC

con lo que representa. Lo simbólico ha dejado de ser pura mediación entre la mente y la objetividad para alcanzar sus propios niveles de realidad, como si hubiese adquirido cartas de ciudadanía, como si Platón resucitara y con él, el mundo de las ideas hipostasiadas.

Por otra parte, la cultura de la inmediatez está operando tanto en el acceso a la información como la expectativa de comprensión inmediata de la realidad o de los textos, lo cual no se logra sin el esfuerzo del discurso pausado y analítico. Las consecuencias en el aprendizaje de esta inmediatez radican en el acceso fraccionado al conocimiento y a la realidad, en los saltos discursivos y en la insuficiencia argumentativa ${ }^{31}$. Desde la óptica del docente que se empeña en educar ${ }^{32}$ el pensamiento por la vía de la conformación de categorías de pensamiento, de desarrollo del pensamiento analítico y crítico y de la comunicación ordenada y sustentada, no deja de ser desesperante toparse con textos sin pies ni cabeza, y me alarma pensar si los míos son así. Pero a la vez, la preocupación académica se centra en reconocer si se han rediseñado nuevas lógicas argumentativas, al amparo de las tecnologías, donde opera no Los analíticos de Aristóteles o la Lógica Simbólica de Irving M. Copy, los libros de cabecera, sino nuevas maneras de razonar, mezclas de emociones, de contextos sociales y de valoraciones morales. ¿Estamos frente al abandono de la lógica formal? O bien, por lo menos, ¿estamos frente a la incorporación de lógicas de tipo Babel, de pensamiento explosivo, lanzado en múltiples direcciones? O bien ¿hemos optado por la formalidad de los equipos y programas, dejando extinguir el contenido profundo de las ideas?

\section{Aparato conformador de imágenes del mundo y la plasticidad cerebral}

Jost Herbig $^{33}$, un epistemólogo cultural, aborda en su libro La evolución del conocimiento científico. Del pensamiento mítico al pensamiento racional el supuesto de que en los últimos diez mil años no se ha dado el caso de que nuestra conformación cerebral se hubiese cambiado, pero que, en cambio, dotados de un aparato formador de imágenes del mundo lo que hacemos los humanos ${ }^{34}$ en los con

\footnotetext{
José Antonio Pérez Tapias. Filosofía y crítica de la cultura. (Madrid: Editorial Trotta, 1995), 129-144.

Edgar Morin, et al., Educar en la era planetaria. (Barcelona: Gedisa, 2006), 25.

Herbig Jost. La evolución del conocimiento cientifico. Del pensamiento mítico al pensamiento racional. (Barcelona: Herder, 1997), 29-31.

34 López-Calva, M. Educación humanista. (México: Ed. Gernika, 2009), 6-7.
} 
tinuos actos de cognición radica en crear sistemas simbólicos a través de los cuales concebimos la realidad. Para dar fundamento a su tesis Herbig efectúa una revisión de las obras mitológicas y pre-científicas de los antiguos griegos, como Homero y los presocráticos, hasta abordar a Aristóteles en su física y astronomía y a otros pensadores posteriores. En su desarrollo destaca, como vía de secuencia histórica y literaria a la noción de causalidad, que parte de la voluntad arbitraria de los dioses, a la que se atribuían los fenómenos naturales, los acontecimientos históricos, la guerra y la paz, los vencedores y los vencidos, lo mismo que los sentimientos y decisiones de los mortales.

Hoy, en la sociedad del conocimiento, de las tecnologías de la comunicación y la información y de la globalización de la cultura ${ }^{35}$, la causalidad está muy alejada de los dioses y, en cambio, la construcción simbólica de nuestro tiempo posee referentes que responden a otras formas de sacralidad por entero tecnológica. Sin embargo, la construcción de la noción de causalidad ha dejado de tener la antigua linealidad entre causa y efecto, acto y potencia o causa primera y causa segunda de la metafísica aristotélica, para dar paso a redes de multi-causalidades de suyo inasibles e incomprensibles, o bien, en el otro extremo, a la negación de la importancia de la causalidad para poner en primer plano de todo análisis la pura percepción fenoménica de la realidad. Dicho de otra manera, importa la utilidad sobre el sentido, la tecnología, sobre los contenidos. Así, consiguientemente, no importa la secuencia entre causa y efecto, el antecedente y el consecuente, sino que, de manera discursiva podemos invertir la secuencia; o bien, descuidando los procesos epistemológicos a través de los cuales los conceptos tienen su génesis, más bien nos atenemos a la semántica práctica de los términos de uso corriente. Las tecnologías han contribuido a que la simplicidad empobrecida de los significados y de las estructuras sintácticas prive sobre los procesos de inferencia validados por la lógica; importa la equivocidad sobre la univocidad de los términos en la medida que siempre podemos aclarar que, por ejemplo, «para mí, el concepto de democracia significa corrupción», frase en la que no se distingue el significado originario del imaginario colectivo.

35 Marshall Mcluhan. La Aldea Global, 1ª edición, (México, 1991), 203. 
Habilidades de pensamiento simbólico: urdimbres

de significado, sociedad y TIC

\section{Cultura de la construcción simbólica y significado unívoco de las tecnologías}

Las tecnologías de la información y la comunicación han obligado al desarrollo de habilidades de creación simbólica ${ }^{36}$ a través de la generación de signos, códigos, significados e íconos en el contexto de significaciones unívocas ${ }^{37}$ que, sin embargo, abren los márgenes de aplicaciones analógicas. En referencia a los significados unívocos, equívocos y analógicos, el primero de los cuales establece una relación entre su signo y su significado, entre el comando y el resultado de su operación, como es el caso de los signos de la lógica o la matemática; en esos casos, no hay motivo de duda: la flecha horizontal, ubicada entre las letras p, q, expresa la implicación si P, entonces $Q$ y no puede significar ninguna otra cosa, ni relaciones de igualdad, de mayor que, ni ambas cosas, solo de $P$ se sigue $Q$.

Las expresiones equívocas se prestan a los malos entendidos, como es el caso del doble sentido con invariables connotaciones sexuales del humor mexicano; es equívoco decir una cosa por la otra o decir de manera ambigua lo que solo por la aclaración del contexto puede quedar esclarecido. Las proposiciones analógicas hacen referencia a realidades que en parte se parecen y en parte se distinguen, como es el caso de los conceptos de la filosofía o de la vida cotidiana ${ }^{38}$. No es el caso de que los conceptos de la ciencia sean analógicos, sino más bien unívocos.

Esta aclaración preliminar es necesaria solo en la medida que la creación simbólica de las tecnologías posee una dimensión unívoca, dado que cada ícono o cada comando ofrecen con precisión el sentido de la operación derivada. Los procedimientos en el manejo de las tecnologías son complejos pero con claridad lineal, sin bien, para la persona que es ya un iniciado, dispone de caminos de atajo y con opciones diversas, pero nunca erráticas ni aleatorias. Esta cultura de la creación simbólica ha alcanzado el diseño publicitario, propagandístico, artístico, editorial, de espacios urbanísticos y arquitectónicos, mediante programas de lo más variado y sofisticado. En esta confusión entre causa y efecto, o bien entre conceptos

36 Miguel A. Romero Morett, Desarrollo de habilidades filosóficas. Un estudio comparativo y transdisciplinar en el campo educativo. (México: Universidad de Guadalajara, 2006), 161.

37 Bordieu, P. and Passeron, J.C., Reproduction in education, society and culture. (Beverly Hills: Sage, 1977), 8.

38 George Lakoff y Johnson Mark, Metáforas de la vida cotidiana. (Madrid: Colección Teorema, 2009), 48. 
equívocos y unívocos han intervenido, paradójicamente, las tecnologías, en la medida que la profusión de información cancela la posibilidad de la discriminación entre datos verdaderos y hechos reales, entre razonamientos rigurosos y falacias seductoras o bien tan solo entre documentos sustentados con aparatos críticos adecuados y textos de doxa. En suma, la distinción que nos ocupa se expresa entre el pensamiento elaborado para efectos de la vida cotidiana y el pensamiento simbólico construido por cada individuo pero en el marco de las cargas de significado de la cultura, uno de cuyos signos relevantes es la virtualidad.

La noción de cultura está asociada a la conformación de redes de símbolos y significados que mediatizan la relación entre el pensamiento y la realidad $^{39}$, en la medida que no es posible aprehender las cosas de manera directa e inmediata sin que medien los signos de la lengua y del habla, el lenguaje visual, los códigos olfativos, el lenguaje de la lógica, la matemática o la estadística.

Bien sabemos que nuestro mundo es fundamentalmente simbólico y que la semiótica ha ido ocupando reductos de superior y creciente alcance en el arte, en los espacios abiertos del urbanismo y los cerrados de la arquitectura y en todos los ámbitos del saber y del hacer social. Nuestro nuevo referente epistemológico se resume en una semiótica de la cultura, donde tiene hoy cabida de privilegio, el mundo de las ciencias y de las tecnologías de la información y la comunicación. Se impone, como parte del abordaje de los temas y problemas de hoy, una epistemología de la virtualidad porque hemos desarrollado nuevos procesos a través de los cuales construimos y concebimos un concepto, una imagen que se sitúa en el imaginario colectivo de las redes y que genera nuevos sistemas de fe, tan indubitables como los sistemas de fe de las religiones tradicionales.

Con ello quiero decir que la realidad entera, en la que estamos insertos, es una urdimbre de mensajes, de códigos, de decodificadores y de efectos que intervienen sobre el comportamiento y sobre la conformación de estructuras cognitivas; como hemos dicho en otros momentos, nuestro pensamiento ha ido asumiendo nuevos procesos de inferencia, nuevas estructuras cognitivas y ha ido conformando nuevos valores, incluso nuevos

39 Adam Kuper, Cultura. La versión de los antropólogos. (Barcelona: Paidós, 2001), 126-127. 
Habilidades de pensamiento simbólico: urdimbres

delitos sociales, como los ataques de los hackers a diversas páginas de internet. Para decirlo de manera coloquial, la cultura de la conversación interpersonal ${ }^{40}$, del encuentro directo, del diálogo vespertino de los habitantes de los poblados rurales, de las historias relatadas una y otra vez sin alcanzar el fastidio jamás, han cedido su lugar a las redes sociales, impersonales, de encuentros fugaces, medios efectivos para la transmisión inmediata de datos, de sucesos y opiniones, y fuente de creencias, como nuevos oráculos de la revelación. A la vez, las redes sociales, que han alcanzado niveles de especialización en la ciencia, la filosofía o el arte, han desarrollado accesos al conocimiento compartido ${ }^{41}$ y a la multiplicación del saber, lo que no se logra por el encuentro interpersonal y directo ni siquiera entre los profesores e investigadores de una misma universidad. En ello figura una cultura de la diversidad pues tienen cabida los especialistas de todos los ámbitos cognitivos, lo mismo en las redes del conocimiento que del desempeño profesional. Unos y otros, como dice el dicho, son como aves del mismo plumaje que vuelan en parvadas.

\section{Cultura de la inmediatez}

Afirma Watson que:

La cultura de la respuesta rápida y la facilidad de acceso a todo están potenciando los errores. Esto está conduciendo a un estado de estupidez.parcial constante y a un caos multitarea. Aunque la multitarea significa que estamos mejorando en pensar rápido, la calidad del pensamiento está sufriendo. Podemos hacer más de una cosa a la vez, pero es raro que las hagamos bien ${ }^{42}$.

No se da el caso de que la totalidad de las multitareas a las que nos vemos obligados dependa en exclusiva de las tecnologías de la comunicación y la información, sino a la vida actual, sobre cargada de acciones pendientes y de estímulos de lo más diverso, pero ¿cómo separar esta vida de las tecnologías, pues mas bien forman una y la misma realidad? Con el pretexto de atender las demandas profesionales las tecnologías facilitan las tareas y, a la vez, nos sumergen en complejos de interacciones simultáneas y fraccionan nuestra atención, cada vez más pendular y menos centrada en el alcance de cometidos puntuales.

40 J. C. Filloux, Intersubjetividad y formación. (Buenos Aires: Novedades Educativas, 1996), 20-21.

41 Edgar Morin, El Método III. El conocimiento del conocimiento. (Madrid: Ediciones Cátedra, 1999), 247-248.

42 Richard Watson, Mentes del futuro. ¿Está cambiando la era digital nuestras mentes?, 16. 
De manera paradójica, el «justo a tiempo» del modelo de producción económica obliga a la entrega de resultados en tiempo y forma, lo cual no se nada fácil a causa de las distracciones de las tecnologías. Pensemos en que muchas de las tareas que debemos efectuar corresponden a las que en su clásico libro Robert J. Reich ${ }^{43}$, El trabajo de las naciones, denominaba acciones de «análisis simbólico», para referirse a aquellas en las que se aplicaban habilidades de abstracción, de trabajo colegiado y de experimentación. ¿Quiénes desarrollan estas habilidades simbólico - analíticas? Los estadistas, los inventores, los escritores, los filósofos, los investigadores, y, claro, los diseñadores y creadores de software y hardware. Para entregar resultados en tiempo y forma, los analistas simbólicos requieren espacios de privilegio, en lo posible, fuera de la opresión de los escenarios laborales tradicionales ${ }^{44}$; ellos se valen de las tecnologías pero no son afectados por sus dispersiones, lo cual revela que el aprendizaje es posible y altamente fecundo ${ }^{45}$.

\section{Cultura de la conectividad constante e inmediata}

Watson recuerda que "las empresas están creando una cultura de la conectividad constante e inmediatez" ${ }^{\prime 46}$, todos los días y todas las horas del año, de tal manera que se abre la posibilidad que de ello se generen daños personales y familiares y se revierta a las empresas mediante demandas. Junto a ello, hay que reconocer que, de todas maneras, por razones personales, estamos conectados siempre, de día y de noche, a través de los celulares o móviles, a través de los cuales recibimos mensajes de internet. Esta cultura de la inmediatez es la que nos hace desesperar cuando el equipo se tarda en abrir o descargar una página o cuando enfrentamos una función errónea del software ${ }^{47}$. Queremos obtener los resultados de manera inmediata, sin reconocer que el acceso veloz a la información no se traduce en un aprendizaje eficaz, en una retención en la memoria de los datos relevantes o pertinentes para el objeto de nuestra investigación ${ }^{48}$. Más aún, esta

Robert J. Reich. El trabajo de las naciones. (Buenos Aires, Vergara. 1993), 8.

44 M. Foucault, Diálogos sobre el poder. (Buenos Aires: Alianza, 1990), 10-15.

45 René Crocker Sagastume, et al., Desarrollo curricular por competencias profesionales integradas. (México: Universidad de Guadalajara, 2005), 46-50.

46 Richard Watson, Mentes del futuro. ¿Está cambiando la era digital nuestras mentes?, 32.

47 George Lakoff y Johnson Mark, Metáforas de la vida cotidiana. (Madrid: Colección Teorema, 2009), 57.

48 María Guadalupe Moreno Bayardo y Miguel Agustín Romero Morett Ética, "Investigación Educativa y Formación de Investigadores: Entre la norma y el proyecto de vida”, Revista Iberoamericana sobre Calidad, Eficacia y Cambio en Educación, vol. 9, No. 2, (2011). 
Habilidades de pensamiento simbólico: urdimbres

de significado, sociedad y TIC

cultura de la inmediatez nos impide la lectura reposada, la reflexión que convierte las ideas en aprendizaje significativo y el procesamiento analítico por el que debatimos con los autores y expresamos un punto de vista sustentado y eficaz. El aprendizaje significativo de las teorías solo puede lograrse a partir de la lectura reposada, del seguimiento argumentativo y de la asimilación narrativa.

\section{Cultura de la multitarea o de las polivalencias}

Cultura de la multitarea se refiere al hecho de que, mediante las tecnologías de la comunicación e información hemos desarrollado habilidades para efectuar diversas tareas de manera simultánea ${ }^{49}$, si bien, sin garantía de que todas las hagamos bien y sin que por ello ingrese información consolidada al pensamiento ${ }^{50}$. Esto es así, no solo a causa de la variedad de estímulos simultáneos, sino a que ninguno de los medios está habilitado para transmitir información completa, adecuadamente procesada y lista para generar pensamiento profundo. Ha quedado muy en el pasado el modelo de producción de las monotareas o de las destrezas únicas ${ }^{51}$, para poner en su lugar las tareas complejas y las multihabilidades; tan solo al personal secretarial de las empresas, mucho más que el dominio taquigráfico, se les pide el manejo de Word, Excel y Power Point, por lo menos.

Para ilustrar con un caso más familiar, a un profesor investigador de universidades públicas en México, las tareas que se le piden exigen dominios en habilidades de pensamiento, de argumentación específicamente, de comunicación oral y escrita, de trato humano, de organización, de gestión, de creación e innovación del conocimiento, de manejo de las tecnologías de comunicación e información, entre otras. Esta gama de habilidades se ubica en el contexto de las tecnologías de información y comunicación a causa de que mediante ellas todas las demás se expresan y concretan, por ejemplo, en la realimentación de tareas de los alumnos, en el llenado de formatos en línea para presentar informes y para gestionar apoyos, o en la

49 Miquel Francés, Nuevos perfiles profesionales en la digitalización de las industrias culturales. TELOS. http://sociedadinformacion.fundacion.telefonica.com/telos/tribunagrabar.asp@idarticulo=2\&rev=67.htm (enero 8 de 2013).

50 Edgar Morin, El Método V. La humanidad de la humanidad. La identidad humana. (Madrid: Ediciones Cátedra, 2003).

51 Jost Herbig, La evolución del conocimiento cientifico. Del pensamiento mitico al pensamiento racional (Barcelona: Herder, 1997), 40. 
presentación de las clases y el dictado de conferencias ${ }^{52}$. La misma gama de posibilidades informáticas asociadas al desempeño profesional o al desarrollo académico es, en sí misma, altamente compleja.

\section{La experiencia docente cotidiana}

La reflexión teórica propuesta se vería incompleta e inconsistente si no pudiera articularla a la experiencia cotidiana en el aula ${ }^{53}$, justamente uno de los sitios de privilegio - no es el único- del aprendizaje. Con base en dicha experiencia docente en los niveles de pregrado y posgrado, lo mismo en los cursos presenciales que en los cursos en línea, se resume de la siguiente manera:

- El aprendizaje fraccionario y fraccionado. La vinculación argumentativa y narrativa interrumpida entre párrafo y párrafo o entre frase y frase. Este comportamiento intelectual se expresa igualmente en otras conductas, como en la escucha de los principios de una canción y en el paso a la siguiente, o en paso de un canal a otro, al no disponer de la paciencia necesaria para mirar un programa completo. En el nivel de comprensión intelectual de un texto escrito o de una disertación oral este aprendizaje fraccionario se expresa en la incapacidad para dar seguimiento al proceso argumentativo y, en cambio, registra datos aislados sin eslabonamiento con los demás, y sin visión sistémica del contenido. Este pensamiento de saltamontes ha generado la falta de concentración. Lomelí López recuer$\mathrm{da}$, siguiendo a otros autores, que

Debido a que Google es el sistema de búsqueda más popular, usado por ocho de cada diez internautas, su papel como «organizador» de la red influye directamente en nuestros bábitos. Por medio de sus sistemas de escaneo y rastreo estimula la lectura fragmentaria y veloz: Y eso tiene implicaciones ${ }^{54}$.

- La localización hábil de textos o de frases textuales, de los autores, que resumen las categorías de pensamiento, no así, de las posturas intelectuales sostenidas por los autores. Los estudiantes registran esas frases clave y valoro que lo hagan pero lamento que no las ubiquen como expresiones del marco más acabado de la teoría.

\footnotetext{
52 P. Vain, “¿Y si el alumno no estuviera allí? Una mirada acerca del rol docente universitario, desde las prácticas de la enseńanza en entornos no presenciales”. (Tesis Doctoral en Universidad de Málaga, 2007).

53 J.C. Filloux, Intersubjetividad y formación. (Buenos Aires: Novedades Educativas, 1996).

54 Lomelí López, "De la letra impresa al byte. ¿Internet está reprogramando nuestro cerebro?, 40.
} 
- La confusión entre la descripción, reproducción o parafraseo de los textos y de las ideas de los autores revisados y los planteamientos analíticos propios; o peor, que asuman como análisis lo que en verdad es tan solo un parafraseo $^{55}$. En este mismo sentido figuran las dificultades para establecer y reconocer la separación de voces, entre la palabra del autor, la palabra de los autores referidos por él y la palabra expresada por los estudiantes en sus trabajos académicos, lo mismo de fin de cursos que de investigación o tesis. Se subraya especialmente la enorme dificultad de analizar, interpretar y proponer, pues requiere pensamiento sistémico, conocimiento de horizontes abiertos y densidad argumentativa, nada de lo cual se desarrolla adecuada y aceptablemente en la educación de todos los niveles.

- En el caso de los cursos presenciales destaca particularmente la dificultad de expresar una idea con una estructura gramatical básica. A ello añadimos la yuxtaposición de ideas y de frases atropelladas que hacen del discurso un continuo sin cortes, sin pausas, pero sin eslabonamiento. La dinámica asociativa entre frases y pensamientos parece remitirnos a una asociación libre de ideas o de emociones. Enseñar a puntualizar, no solo en el texto, sino en el habla y en la argumentación, constituye un pendiente en el que debemos trabajar insistentemente.

- Las dificultades de concentración. En este aspecto, Watson nos recuerda que "las interrupciones digitales constantes y el exceso de información están atomizando nuestra atención y destruyendo nuestra concentración. Nos resulta difícil concentrarnos y nos estamos volviendo adictos a la pantalla. Debemos alejarnos de la idea de que toda información es útil y ajustarnos a una realidad nueva en el que la atención es poder y lo más crítico es la confianza en la información" ${ }^{36}$. En este aspecto la crítica a la confianza en la información, como fuente indubitable, tiene su correlato en la confianza en la realidad a la que la fenomenología considera como la más insidiosa de las creencias. Me refiero a considerar que la realidad está por entero hecha y que su significado está por entero previsto desde aquella. Volvamos a las cosas mismas, al hombre mismo, a los valores ${ }^{57}$ originarios y originantes, hagamos epojé, la saludable suspensión del jui-

55 P, Bourdieu y J. C. Passeron, La reproducción: elementos para una teoría del sistema de enseñanza. (Madrid: Popular, 2001), 62.

56 Richard Watson, Mentes del futuro. ¿Está cambiando la era digital nuestras mentes?, 32.

57 F. Savater, El valor de educar. (México: Ariel 1997), 70. 
cio que nos hace escapar de los supuestos acríticos, de las conclusiones anticipadas y de las inferencias que exceden los alcances de sus premisas.

- Perseverar obstinadamente en el área de luminiscencia y postergar o rechazar el ingreso al área de umbriscencia. La luz es confort y seguridad, pero contrario a lo que suponemos, en ella no está el conocimiento, sino en lo umbrío, lo soterrado, lo obscuro, lo desconocido, lo extraño, lo nuevo. Los estudiantes de los cursos en línea, que ya poseen una formación preliminar en su carrera profesional primera y en su camino laboral andado, insisten en hacer referencia a sus dominios cognitivos familiares, estableciendo forzadas analogías entre su saber antiguo y el saber nuevo propuesto en los cursos. Sus referentes cognitivos no cambian, si bien, algunos logran exitosamente el pensamiento analógico entre ámbitos cognitivos diversos. Este salto analógico constituye una perspectiva educativa que debe fomentarse.

- La resistencia a la memorización de datos, frases, hechos, fechas, ante el supuesto de que toda la información está en las bases de datos o en la red, olvidando que sabemos solo lo que en verdad recordamos. Ha sido una mala medida minusvalorar el saber de la memoria y suplirlo por la habilidad de manejo de la tecnología para buscar información. De hecho debemos incorporar estas dos habilidades a una tercera, la de pensamiento analítico y crítico.

- El uso inadecuado o insuficiente de las Tecnologías de Información y Comunicación para usos de aprendizaje significativo.

\section{CONCLUSIÓN}

\section{Cómo implementar las TIC en Educación}

La cuestión fundamental de la relación entre las TIC y la educación no radica en si debemos integrarlas o no o en qué medida, pues es claro que debemos hacerlo, sino integrarlas de tal manera que se articulen al proceso de enseñanza, en cuestión, un aprendizaje para generar formas de pensamiento profundo. Aunque la mayoría de los profesores siguen apegados al modelo tradicional, en el que continúan fieles al paradigma de la educación tradicional y únicamente utilizan los libros como herramienta para que el 
Habilidades de pensamiento simbólico: urdimbres

de significado, sociedad y TIC

alumno pueda adquirir conocimientos según el modelo de educación presencial. Aún las tecnologías son empleadas como extensiones del mismo modelo tradicional, en la medida que las interacciones quedan reducidas a un solo sentido.

Tampoco se debe de caer en la falsa creencia que únicamente con digitalizar los libros o los diferentes recursos que se utilizan como herramientas en la clase, se virtualiza el método de enseñanza, pues ello se traduce simplemente es cambiar el formato físico por uno más transportable y más cómodo. De hecho, el uso de videos, diapositivas, animaciones o internet no equivale al aprovechamiento de las potencialidades pedagógicas de las tecnologías. Nuevamente se insiste en que el reto real se sitúa en dinámicas de interacción en red, en la construcción colegiada del saber y en la apropiación del aprendizaje significativo.

Todos estos formatos y tecnologías digitales no funcionan por el simple hecho de ser nuevos, es indispensable que el profesor también continúe su proceso impartiendo una educación de calidad y no el mismo contenido utilizado por años en un nuevo formato digital. El primer paso que debemos tomar es capacitar a nuestros profesores en el uso de las TIC, ya que sin esta capacitación será muy difícil que ellos mismos las utilicen, y a la vez puedan y sepan diferenciar entre la educación con el apoyo de las TIC y la enseñanza de las mismas.

Además, las universidades no deberán de limitar las ideas y la elección de materiales a utilizarse con estas tecnologías, con base al contenido de la asignatura, teniendo la libertad de hacer estas elecciones, los profesores podrán participar en comunidades virtuales especializadas donde podrán intercambiar diversos recursos del área académica correspondiente.

De cualquier manera y como una insistencia final y congruente con los planteamientos iniciales, la atención debe centrarse en los aspectos vinculados a la argumentación, al pensamiento profundo, causal y simbólico, a la comprensión de textos de mediana y alta complejidad, a la producción de textos literarios, científicos y filosóficos. Ante la aparente distancia de una formación de este tipo causa una gran preocupación de los estudian- 
tes, el alcance de los nichos de mercado y la incertidumbre laboral ${ }^{58}$. Hay en ello una perversión de visión, como lo hay en el sentido de optar por la forma de la estructura material del hardware y conceptual del software; las habilidades, a la vez tecnológicas y cognoscitivas obedecen a la cotidiana inquietud académica y docente que nos enfrenta a un escenario de incertidumbre en el mundo laboral. Los jóvenes siguen buscando su formación y muchos de ellos perseveran en su convencimiento de que la educación, las artes, las humanidades, la filosofía, las ciencias experimentales, formales y sociales constituyen un horizonte de crecimiento profesional y de vida ${ }^{59}$. Sin embargo, sin las habilidades simbólicas - analíticas toparán con pared. Todas las ciencias y las tecnologías requieren de este tipo de habilidades de pensamiento, es decir, de habilidades de abstracción, de resolución de problemas, de experimentación y de trabajo en equipo que los escenarios laborales ${ }^{60}$.

\section{REFERENCIAS}

Aguilar, Luis Armando (coord.); Keiner, Dieter; Mejía Aruz, Rebeca; Morán Quiroz, Luis Rodolfo, y Romero Morett, Miguel Agustín. Lo bumano en riesgo. Educación frente a la globalización. Instituto Tecnológico de Occidente. México: Jalisco, 2005.

Asociación Mexicana de Internet. Estudios | Asociación Mexicana de Internet. Estudios | Asociación Mexicana de Internet.

http:/ / www.amipci.org.mx/temp/Habitos2011AMIPCI-VersionEjecutiva-000834 3001306794341OB.pdf.

Asociación Mexicana de Internet. Asociación Mexicana de Internet. Obtenido de Asociación Mexicana de Internet. www.amipci.org.mx.

Barnett, Roland. Los límites de la competencia. España: Editorial Gedisa, 2001.

Bartra, Roger. Antropología del cerebro. La conciencia y los sistemas simbólicos. FCE. México. ISBN 13: 97884254193242012.

Beneitone, Pablo, et al., Reflexiones y perspectivas de la educación superior en América Latina. Informe final - Proyecto Tuning - América Latina, 2004-2007. España: Universidad de Deusto, 2007.

Brea, José Luis (editor). Estudios visuales. La epistemología de la visualidad en la era de la globalización. Madrid: Akal, 2005.

\footnotetext{
58 A. Catalano, et al., Diseño curricular basado en normas de competencia laboral: conceptos y orientaciones metodológicas. (Buenos Aires: BID/FOMIN, Cinterfor, 2004), 226.

59 Edgar Morin, El Método V. La humanidad de la humanidad. La identidad humana. (Madrid: Ediciones Cátedra, 2003), 280

60 A. Catalano, et al., Diseño curricular basado en normas de competencia laboral, 226.
} 
Habilidades de pensamiento simbólico: urdimbres de significado, sociedad y TIC

Briasco, I.; Vargas, F. La implementación de sistemas por competencias: impacto en el modelo de gestión organizacional. Experiencias y estrategias para la acción. Documento presentado en el Foro Iberoamericano sobre Educación y Trabajo. Área temática: Formación de técnicos y profesionales competentes: un futuro deseable. Febrero, 2001.

Bourdieu, P. y Passeron, J. C. La reproducción: elementos para una teoría del sistema de enseñanza. Madrid: Popular, 2001.

Bordieu, P. and Passeron, J. C. Reproduction in education, society and culture. Beverly Hills: Sage, 1977.

Catalano, A. Ma.; Avolio de Cols, S.; Sladogna, M. G. Diseño curricular basado en normas de competencia laboral: conceptos y orientaciones metodológicas. Buenos Aires: BID/FOMIN; Cinterfor, 2004.

Crocker Sagastume, René, et al. Desarrollo curricular por competencias profesionales integradas. México: Universidad de Guadalajara, 2005.

Escámez, J. "Actitudes en Educación”, en Altarejos, F. y otros. Filosofía de la educación boy. Madrid: Dykinson, 1991, 525-539.

Francés, Miquel. Nuevos perfiles profesionales en la digitalización de las industrias culturales. Página: TELOS, 2013.

http://sociedadinformacion.fundacion.telefonica.com/telos/tribunagrabar.asp@ idarticulo $=2 \& \mathrm{rev}=67 . \mathrm{htm}$

Flaschsland, C. Pierre Bourdieu y el capital simbólico. Buenos Aires: Campo de Ideas, 2005.

Foucault, M. Diálogos sobre el poder. Buenos Aires: Alianza, 1990.

Filloux, J. C. Intersubjetividady formación. Buenos Aires: Novedades Educativas, 1996.

Ferry, G. Pedagogía de la formación. Argentina: Novedades Educativas, 1997.

Giménez Montiel, Gilberto. "Teoría y análisis de la cultura”. Revista Conaculta. Intersecciones. Vols. I, II, (2005).

Galeano, E. Patas arriba. La escuela del mundo al revés. Buenos Aires: Siglo XXI, 1998.

Geertz, C. Old societies and new states. The quest for modernity in Asia and Africa. New York: The Free Press, 1963.

Hadot, Pierre. ¿Qué es la filosofía antigua? México: Fondo de Cultura Económica, 1998.

Herbig, Jost. La evolución del conocimiento cientifico. Del pensamiento mítico al pensamiento racional Barcelona: Herder, 1997.

Honoré, B. Para una teoría de la formación. Madrid: Narcea, 1980.

Kuper, Adam. Cultura. La versión de los antropólogos. Barcelona: Paidós, 2001.

Lakoff, George y Johnson, Mark. Metáforas de la vida cotidiana. Madrid. Colección Teorema, 2009.

Lomelí López, Jonathan. "De la letra impresa al byte. ¿Internet está reprogramando nuestro cerebro?”. Magis. Revista del ITESO. Diciembre 2012 - Enero 2013/ Año XLII número (2012 - 2013): 38-44.

Lévy, Pierre. ¿Qué es lo virtual? Barcelona: Paidós, 1999. 
López-Calva, M. Educación humanista. México: Ed. Gernika, 2009.

Moreno Bayardo, María Guadalupe y Romero Morett, Miguel Agustín. “Ética, Investigación Educativa y Formación de Investigadores: Entre la norma y el proyecto de vida". Revista Iberoamericana sobre Calidad, Eficacia y Cambio en Educación, vol. 9, No. 2, (2011).

Mcluban, Marshall. La Aldea. Global, P. Baricco, Alessandro. 1a edición, México, 1991.

Morin, Edgar. El Método II. La vida de la Vida. Madrid: Ediciones Cátedra, 1997.

Morin, Edgar. El Método III. El conocimiento del conocimiento. Madrid: Ediciones Cátedra, 1999.

Morin, Edgar. Los siete saberes necesarios para la educación del futuro. Buenos Aires: Ed. Nueva Visión, 2001.

Morin, Edgar. El Método V. La humanidad de la humanidad. La identidad humana. Madrid: Ediciones Cátedra, 2003.

Morin, E.; Roger., E. y Motta., R. Educar en la era planetaria. Primera reimpresión. Barcelona: Gedisa, 2006.

Pérez Tapias, José Antonio. Filosofía y crítica de la cultura. Madrid: Editorial Trotta, 1995.

Reich, Robert J. El trabajo de las naciones. Buenos Aires: Vergara, 1993.

Reynoso, Carlos. Corrientes teóricas en antropología. Perspectivas desde el siglo XXI. SB Argentina: Buenos Aires, 2008.

Romero Morett, Miguel A. Desarrollo de habilidades filosóficas. Un estudio comparativo y transdisciplinar en el campo educativo. México: Universidad de Guadalajara, 2006.

Savater, F. El valor de educar. México: Ariel, 1997.

Shulman, L. Knowledge and teaching: foundations of the new reform. Harvard Educational Review, 1987, 57 (1).

Solares, Blanca (coord.). Los lenguajes del símbolo. Investigaciones de hermenéutica simbólica. UNAM: Anthropos, 2001.

Vain, P. ¿Y si el alumno no estuviera alli? Una mirada acerca del rol docente universitario, desde las prácticas de la enseñanza en entornos no presenciales. Tesis Doctoral en Universidad de Málaga. 2007.

Watson, Richard. Mentes del futuro. ¿Está cambiando la era digital nuestras mentes? Viceversa ayer y hoy, Barcelona, 2011.

Zapata Zapata, Donna. Contextualización de la enseñanza virtual en la educación superior. Bogotá: ICFES, 2002.

http://blade1.uniquindio.edu.co/uniquindio/ntic/lineamientos/nuevos $\% 20$ libros/arc_88. pdf.

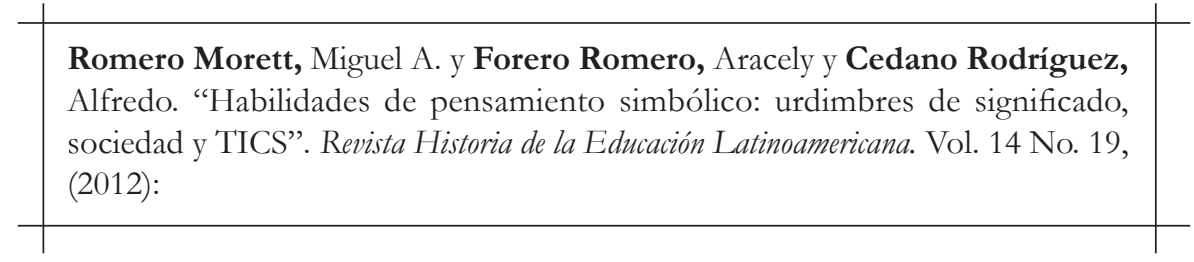

